\title{
Ausschreibung „Bibliothek des Jahres 2021“ sowie „Bibliothek des Jahres in kleinen Kommunen und Regionen 2021“
}

https://doi.org/10.1515/bd-2021-0030

Der Deutsche Bibliotheksverband e.V. (dbv) und die Deutsche Telekom Stiftung schreiben die Auszeichnung „Bibliothek des Jahres 2021“ aus. Damit wird am 24. Oktober 2021 - dem „Tag der Bibliotheken“ - zum 22. Mal der Preis „Bibliothek des Jahres“ verliehen. Die Auszeichnung ist der einzige nationale Bibliothekspreis in Deutschland.

Mit der Auszeichnung soll ein Beitrag zum Image der Bibliotheken in der digitalen Welt geleistet werden. Sie würdigt Bibliotheken aller Sparten und ausdrücklich auch aller Größen. Prämiert wird vorbildliche und innovative Bibliotheksarbeit unter Nutzung von digitalen Angeboten und Services. Der Preis ist mit 20.000 Euro dotiert.

\section{Bibliothek des Jahres in kleinen Kommunen und Regionen}

Zum zweiten Mal wird in diesem Jahr zusätzlich die Auszeichnung „Bibliothek des Jahres in kleinen Kommunen und Regionen“ für innovative Bibliotheksarbeit und regionales Engagement kleinerer Bibliotheken verliehen. Die Auszeichnung würdigt Bibliotheken aller Sparten in Städten und Gemeinden mit bis zu 50.000 Einwohner*innen. Der Preis ist mit 7.000 Euro dotiert.

Vorschlagsberechtigt für beide Preise sind Bibliotheksleitungen, die Vorsitzenden der Sektionen, Landesverbände und Kommissionen des dbv sowie die Jurymitglieder.

Einsendeschluss für die Bewerbungen ist der 31.03.2021.

Eine Übersicht über die bisherigen Preisträger-Bibliotheken finden Sie hier: https://www.bibliotheksverband.de/dbv/auszeichnungen/bibliothek-des-jahres/ preistraeger.html.

\section{Auszeichnung Bibliothek des Jahres}

Die Auszeichnung „Bibliothek des Jahres“ ist der einzige nationale Bibliothekspreis in Deutschland und wird gemeinsam vom Deutschen Bibliotheksverband und der Deutsche Telekom Stiftung vergeben. Mit der Auszeichnung soll ein Beitrag zum Image der Bibliotheken in der digitalen Welt geleistet werden. Der Preis ist mit 20.000 Euro dotiert. Er würdigt Bibliotheken aller Sparten und Größen. Zum zweiten Mal wird 2021 zusätzlich eine Bibliothek in einer Kommune 
oder Region mit bis zu 50.000 Einwohner*innen ausgezeichnet. Der Preis ist mit 7.000 Euro dotiert.

Weitere Informationen unter: https://www.bibliotheksverband.de/dbv/ auszeichnungen/bibliothek-des-jahres.html.

\section{Der Deutsche Bibliotheksverband e.V. (dbv)}

Der Deutsche Bibliotheksverband e.V. (dbv) vertritt mit seinen mehr als 2.100 Mitgliedern bundesweit über 9.000 Bibliotheken mit 25.000 Beschäftigten und 11 Mio. Nutzer^innen. Sein zentrales Anliegen ist es, Bibliotheken zu stärken, damit sie allen Bürger^innen freien Zugang zu Informationen ermöglichen. Der Verband setzt sich ein für die Entwicklung innovativer Bibliotheksleistungen für Wissenschaft und Gesellschaft. Als politische Interessensvertretung unterstützt der dbv die Bibliotheken insbesondere auf den Feldern Informationskompetenz und Medienbildung, Leseförderung und bei der Ermöglichung kultureller und gesellschaftlicher Teilhabe für alle Bürger^innen.

Mehr Informationen finden Sie unter www.bibliotheksverband.de.

\section{Die Deutsche Telekom Stiftung}

Die Deutsche Telekom Stiftung wurde 2003 gegründet, um den Bildungs-, Forschungs- und Technologiestandort Deutschland zu stärken. Mit einem Kapital von 150 Millionen Euro gehört sie zu den großen Unternehmensstiftungen in Deutschland. Sie konzipiert und realisiert Projekte, die sich mit Themen aus dem mathematisch-naturwissenschaftlich-technischen Umfeld beschäftigen. Die Stiftung setzt sich dafür ein, dass junge Menschen in einem Bildungs-Ökosystem lernen, und initiiert und fördert daher Kooperationen verschiedener Lernorte.

Mehr Informationen finden Sie unter www.telekom-stiftung.de.

\section{Pressekontakte:}

Kristin Bäßler

Deutscher Bibliotheksverband

Leitung Kommunikation / Pressesprecherin

Tel.: + 49 (0)30 6449899 25 | E-Mail: baessler@bibliotheksverband.de

Matthias Adam

Deutsche Telekom Stiftung

Kommunikation

Tel. +49 (0)228 18192015 | E-Mail: matthias.adam@telekom-stiftung.de 